\title{
Dinitrogen Activation by Titanium Sandwich Complexes.
}

\author{
Tamara E. Hanna, Emil Lobkovsky and Paul J. Chirik* \\ Department of Chemistry and Chemical Biology, Baker Laboratory, Cornell University, \\ Ithaca, New York 14853 \\ Supporting Information
}




\section{Table of Contents}

$\begin{array}{ll}\text { React-IR data for } \mathrm{N}_{2} \text { binding to } 4 & \text { S3 }\end{array}$

$\begin{array}{ll}\text { React-IR data for } \mathrm{N}_{2} \text { binding to } 5 & \mathrm{~S} 4\end{array}$

$\begin{array}{ll}\text { Experimental details } & \text { S5 }\end{array}$

$\begin{array}{ll}\text { X-ray crystallographic data for } \mathbf{6}-\left(\mathbf{N}_{2}\right)_{2} & \text { S12 }\end{array}$

$\begin{array}{ll}\text { References } & \text { S16 }\end{array}$ 


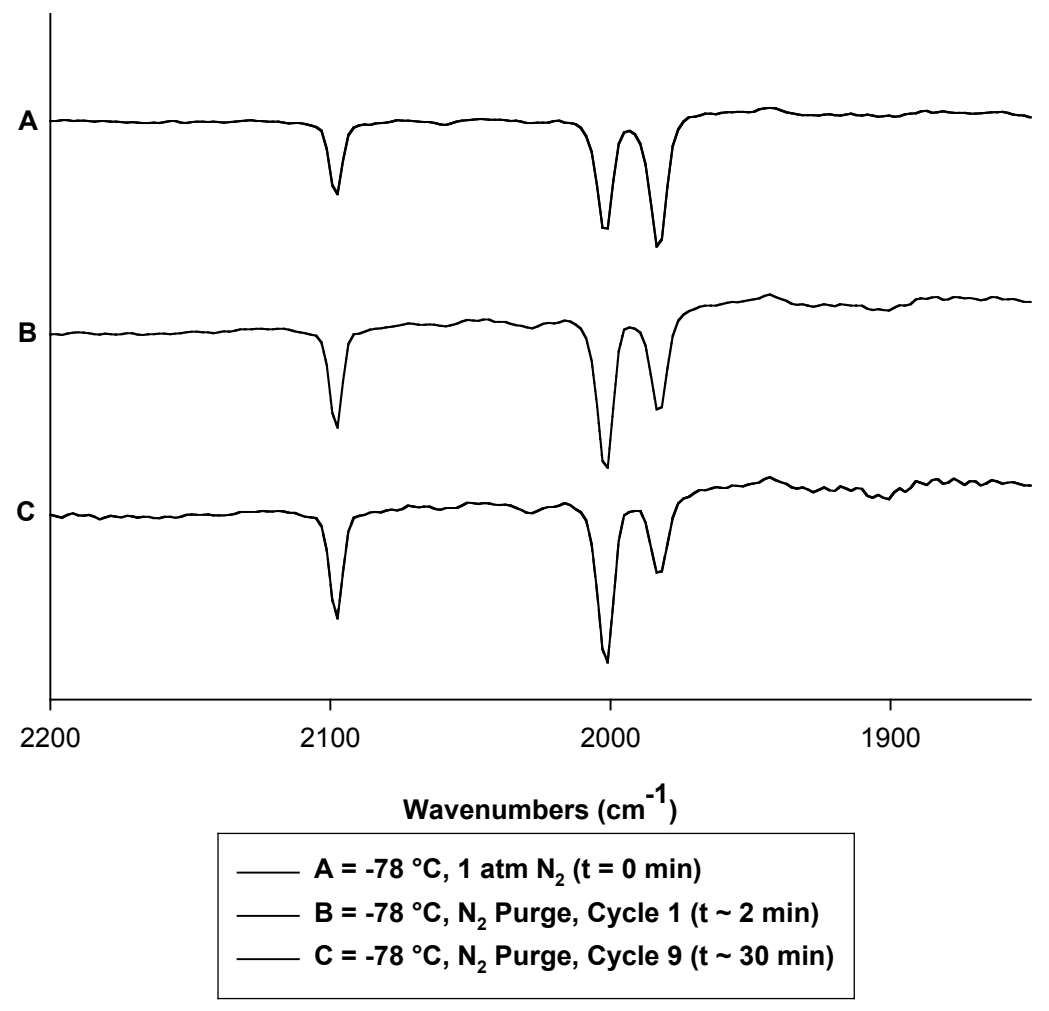

Figure S1. React-IR spectrum of the reaction of $\mathbf{4}$ with dinitrogen in pentane solution. 


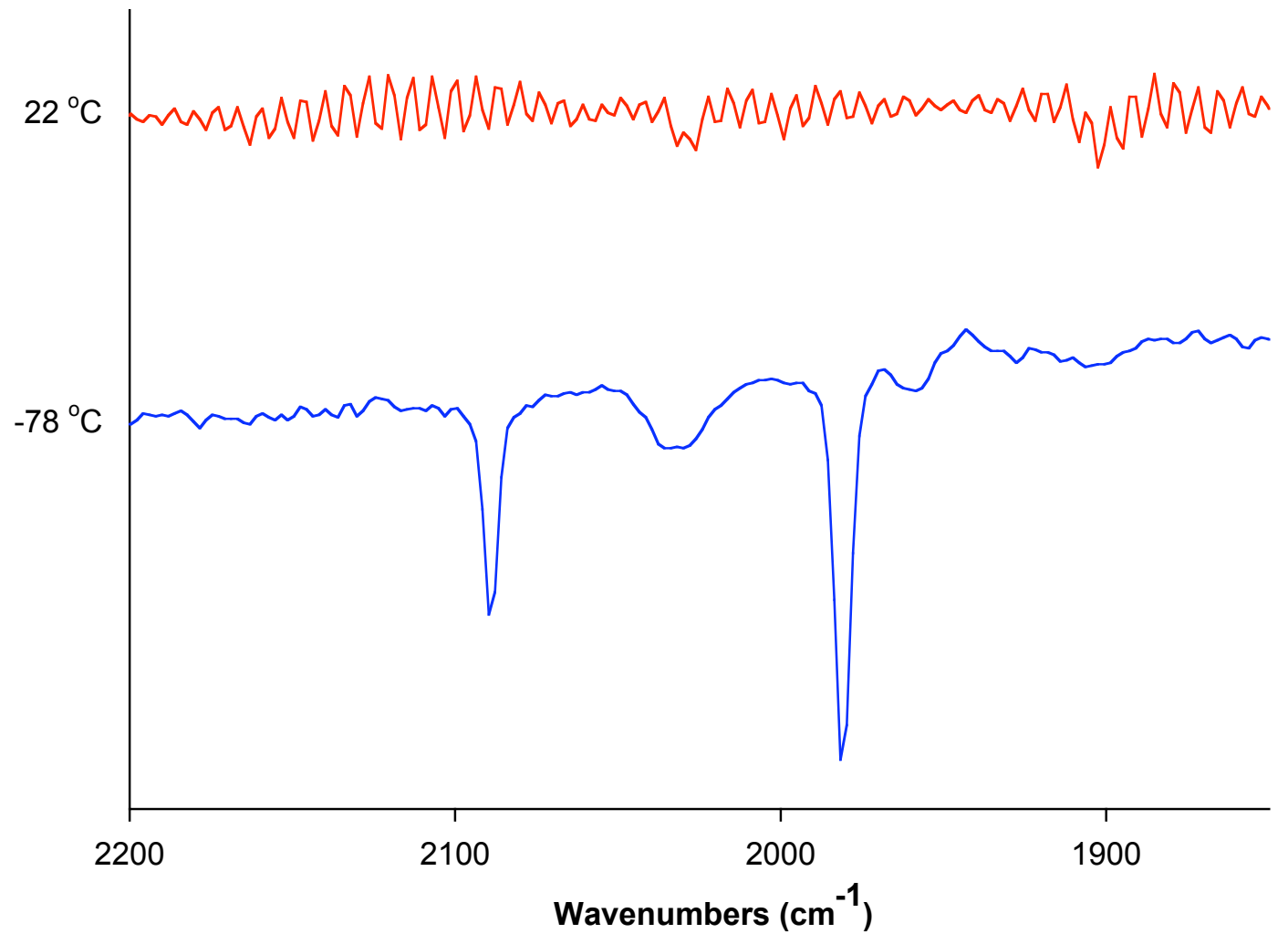

Figure S2. React-IR spectrum of the reaction of $\mathbf{5}$ with dinitrogen in pentane solution. 


\section{Experimental Section}

General Considerations. All air- and moisture-sensitive manipulations were carried out using standard high vacuum line, Schlenk or cannula techniques or in an M. Braun inert atmosphere drybox containing an atmosphere of purified nitrogen. The M. Braun drybox was equipped with a cold well designed for freezing samples in liquid nitrogen. Solvents for air- and moisture-sensitive manipulations were dried and deoxygenated using literature procedures. ${ }^{1}$ Deuterated solvents for NMR spectroscopy were purchased from Cambridge Isotope Laboratories and were distilled from sodium metal under an atmosphere of argon and stored over $4 \AA$ A molecular sieves. Hydrogen and argon gas were purchased from Airgas Incorporated and passed through a column containing manganese oxide supported on vermiculite and $4 \AA$ molecular sieves before admission to the high vacuum line. Carbon monoxide was passed through a liquid nitrogen cooled trap immediately before use. Both $\mathrm{Li}\left[\mathrm{C}_{5} \mathrm{Me}_{4} \mathrm{CMe}_{3}\right]^{2}$ and $\left(\square^{5}-\mathrm{C}_{5} \mathrm{Me}_{4} \mathrm{SiMe}_{3}\right)_{2} \mathrm{TiCl}_{2}^{3}$ were prepared according to literature procedures. Titanocene $\mathbf{4}$ was prepared in analogy to $\mathbf{5}$ (vide infra) and was characterized by comparison to literature data. ${ }^{4} \mathrm{C}_{5} \mathrm{Me}_{4} \mathrm{H}\left(\mathrm{CHMe}_{2}\right)$ was prepared in analogy to $\mathrm{C}_{5} \mathrm{Me}_{4} \mathrm{H}\left(\mathrm{CMe}_{3}\right)^{5}$ and was identified by comparison to reported spectral data. $^{5}$

${ }^{1} \mathrm{H}$ spectra were recorded on Varian Mercury 300, Inova 400 and 500 spectrometers operating at 299.763, 399.780 and $500.62 \mathrm{MHz}$, respectively. All chemical shifts are reported relative to $\mathrm{SiMe}_{4}$ using ${ }^{1} \mathrm{H}$ (residual) chemical shifts of the solvent as a secondary standard. Magnetic moments were measured at $22{ }^{\circ} \mathrm{C}$ by the method originally described by Evans ${ }^{6}$ with stock and experimental solutions containing a known amount of a ferrocene standard. 
Single crystals suitable for X-ray diffraction were coated with polyisobutylene oil in a drybox and were quickly transferred to the goniometer head of a Siemens SMART CCD Area detector system equipped with a molybdenum X-ray tube $(\square=0.71073 \mathrm{~A})$. Preliminary data revealed the crystal system. A hemisphere routine was used for data collection and determination of lattice constants. The space group was identified and the data were processed using the Bruker SAINT program and corrected for absorption using SADABS. The structures were solved using direct methods (SHELXS) completed by subsequent Fourier synthesis and refined by full-matrix least-squares procedures. Elemental analyses were performed at Robertson Microlit Laboratories, Inc., in Madison, NJ.

Solution infrared spectra were recorded with an in situ IR spectrometer fitted with a 30-bounce, silicon-tipped probe optimized for sensitivity. The spectra were acquired in 16 scans ( $30 \mathrm{~s}$ intervals) at a gain of one and a resolution of four. A representative reaction was carried out as follows: The IR probe was inserted through a nylon adapter and O-ring seal into a flame-dried, cylindrical flask fitted with a magnetic stir bar and Tjoint. The T-joint was capped by a septum for injections and a nitrogen line. Following evacuations under full vacuum and flushing with nitrogen, the flask was charged with pentane and a background was recorded at ambient temperature and at $-78^{\circ} \mathrm{C}$. The flask was then charged with a pentane solution of titanocene to make the final reaction volume $10.0 \mathrm{~mL}$ with approximately $0.005 \mathrm{M}$ concentration. The samples were cooled using ethanol/dry ice and acetone/dry ice baths and the temperatures were monitored using an external, low-temperature thermometer. The reactions were recorded between 20 and 40 intervals. 
The maximum temperature for $\mathrm{N}_{2}$ coordination was determined by monitoring the IR spectrum as a function of temperature. The temperature of the sample was raised in five degree increments until the $\mathrm{N}_{2}$ stretches were no longer observed. Once the approximate binding temperature was recorded, the temperature of the sample was then changed in approximately one degree increments until a more precise value was measured. The error associated with the measurements is $+/-2{ }^{\circ} \mathrm{C}$. Solid state magnetic measurements were determined with a Johnson-Mathey Mark 1 Magnetic Susceptibility Balance with a voltage of $12 \mathrm{~V}$ DC and a current of $300 \mathrm{~mA}$.

Preparation of $\left(\square^{5}-\mathrm{C}_{5} \mathrm{Me}_{4} \mathrm{SiMe}_{3}\right)_{2} \mathrm{Ti}(\mathrm{CO})_{2}\left(4-(\mathrm{CO})_{2}\right)$. A thick-walled glass vessel was charged with $3.62 \mathrm{~g}$ of $0.5 \%$ (by weight) sodium amalgam and approximately $10 \mathrm{~mL}$ of pentane. With vigorous stirring, $0.77 \mathrm{~g}(0.152 \mathrm{mmol})$ of $\left(\square^{5}-\mathrm{C}_{5} \mathrm{Me}_{4} \mathrm{SiMe}_{3}\right)_{2} \mathrm{TiCl}_{2}$ was added as a pentane solution and the vessel was brought out of the drybox and was immediately frozen in liquid nitrogen. On the high-vacuum line, the vessel was degassed and 1 atm of carbon monoxide was added at $-196{ }^{\circ} \mathrm{C}$. The vessel was thawed and the reaction stirred for 18 hours at ambient temperature. The solvent was removed in vacuo and the reaction was brought into the drybox, filtered through a pad of Celite and recrystallized from pentane to yield $0.060 \mathrm{~g}(80.5 \%)$ of $4-(\mathbf{C O})_{2}$. Anal. Calcd for $\mathrm{C}_{26} \mathrm{H}_{42} \mathrm{O}_{2} \mathrm{Si}_{2} \mathrm{Ti}: \mathrm{C}, 63.64 ; \mathrm{H}, 8.63$. Found: C, 63.37; H, 8.76. ${ }^{1} \mathrm{H}$ NMR (benzene- $d_{6}$ ): $\square 0.32$ $\left(\mathrm{s}, 18 \mathrm{H}, \mathrm{C}_{5} \mathrm{Me}_{4} \mathrm{SiMe}_{3}\right), 1.60\left(\mathrm{~s}, 12 \mathrm{H}, \mathrm{C}_{5} \mathrm{Me}_{4} \mathrm{SiMe}_{3}\right), 1.76\left(\mathrm{~s}, 12 \mathrm{H}, \mathrm{C}_{5} \mathrm{Me}_{4} \mathrm{SiMe}_{3}\right) .{ }^{13} \mathrm{C}$ NMR (benzene- $\left.d_{6}\right): \square 2.24\left(\mathrm{SiMe}_{3}\right), 11.40,14.24(\mathrm{Me}), 93.71,109.37,111.00(\mathrm{Cp})$, 262.06 (CO). IR (pentane): $\square, 1865,1941 \mathrm{~cm}^{-1}(\mathrm{CO})$. 
Observation of $\left(\square^{5}-\mathrm{C}_{5} \mathrm{Me}_{4} \mathrm{SiMe}_{3}\right)_{2} \mathrm{Ti}\left(\mathbf{N}_{2}\right)_{2}\left(4-\left(\mathbf{N}_{2}\right)_{2}\right)$. A React IR cell was charged with $0.024 \mathrm{~g}(0.055 \mathrm{mmol})$ of $\mathbf{4}$ and dissolved in a total of $10 \mathrm{~mL}$ of pentane and injected against a flow of dinitrogen. The cell was cooled to $-78^{\circ} \mathrm{C}$ and a spectrum was recorded under a flow of dinitrogen. The temperature of binding was determined based on recording spectra at different temperatures and observation of the N-N stretches. IR (pentane, $\left.-78{ }^{\circ} \mathrm{C}\right)$ : $\square, 2002,2098 \mathrm{~cm}^{-1}\left(\mathrm{~N}_{2}\right)$.

Preparation of $\left(\square^{5}-\mathbf{C}_{5} \mathbf{M e}_{4}{ }^{t} \mathrm{Bu}\right)_{2} \mathbf{T i C l}(5-\mathbf{C l})$. A $100 \mathrm{~mL}$ round-bottomed flask was charged with $1.14 \mathrm{~g}(6.19 \mathrm{mmol})$ of $\mathrm{Li}\left[\mathrm{C}_{5} \mathrm{Me}_{4}{ }^{t} \mathrm{Bu}\right]$ and $1.15 \mathrm{~g}(3.10 \mathrm{mmol})$ of $\mathrm{TiCl}_{3}(\mathrm{THF})_{3}$ and a reflux condenser and $180^{\circ}$ needle valve were attached. On the high vacuum line, 50 $\mathrm{mL}$ of tetrahydrofuran were added by vacuum transfer and the mixture was allowed to warm to ambient temperature with stirring. The reaction mixture was then refluxed for 60 hours, upon which time the solvent was removed in vacuo, leaving a dark solid. The assembly was transferred to the drybox and the desired product extracted into pentane and filtered through Celite. The pentane was removed in vacuo leaving a blue solid. Recrystallization from pentane resulted in $0.90 \mathrm{~g}(66.3 \%)$ of 5-Cl. Anal. Calcd for $\mathrm{C}_{26} \mathrm{H}_{42} \mathrm{ClTi}$ : C, 71.31; H, 9.67. Found: C, 70.97; H, 9.49. ${ }^{1} \mathrm{H}$ NMR (benzene- $d_{6}$ ): $\square-2.4$ $\left(\mathrm{CD}_{1 / 2}=714 \mathrm{~Hz}\right), 6.3\left(\mathrm{G \square}_{1 / 2}=466 \mathrm{~Hz}\right)$. Magnetic susceptibility (benzene- $\left.d_{6}\right): \square_{\text {eff }}=1.48$ $\square_{\mathrm{B}}$. EPR (toluene): $g=1.96$.

Preparation of $\left(\square^{5}-\mathbf{C}_{5} \mathbf{M e}{ }_{4}{ }^{t} \mathbf{B u}\right)_{2} \mathbf{T i}(\mathbf{5})$. A $100 \mathrm{~mL}$ round-bottomed flask was charged with $16.80 \mathrm{~g}$ of $0.5 \%$ sodium amalgam and approximately $15 \mathrm{~mL}$ of toluene. With vigorous stirring, $0.303 \mathrm{~g}(0.692 \mathrm{mmol})$ of $\mathbf{5 - C l}$ dissolved in toluene was added and the resulting 
reaction mixture was stirred for two days at ambient temperature. The red solution was filtered through Celite and the toluene was removed in vacuo, leaving a red solid. Recrystallization from pentane afforded $0.197 \mathrm{~g}(70.9 \%)$ of 5. Anal. Calcd for $\mathrm{C}_{26} \mathrm{H}_{42} \mathrm{Ti}$ : C, 77.59; H, 10.52. Found: C, 77.68; H, 10.14. ${ }^{1} \mathrm{H}$ NMR (benzene- $d_{6}$ ): $\square 4.03$ (br s, 18H, $\left.\mathrm{C}_{5} \mathrm{Me}_{4}{ }^{t} \mathrm{Bu}\right), 34.11$ (br s, $12 \mathrm{H}, \mathrm{C}_{5} M e_{4}{ }^{\mathrm{t}} \mathrm{Bu}$ ), 62.87 (br s, $12 \mathrm{H}, \mathrm{C}_{5} M e_{4}{ }^{\mathrm{t}} \mathrm{Bu}$ ). Magnetic susceptibility (benzene- $d_{6}, 23^{\circ} \mathrm{C}$ ): $\square_{\mathrm{eff}}=2.58 \square_{\mathrm{B}}$.

Preparation of $\left(\square^{5}-\mathrm{C}_{5} \mathrm{Me}_{4}{ }^{\mathrm{t}} \mathrm{Bu}\right)_{2} \mathrm{Ti}(\mathrm{CO})_{2}\left(5-(\mathrm{CO})_{2}\right)$. A J. Young NMR tube was charged with $0.015 \mathrm{~g}(0.037 \mathrm{mmol})$ of 5 and approximately $0.5 \mathrm{~mL}$ of benzene- $d_{6}$. On the high vacuum line, the tube was degassed and 1 atm of carbon monoxide was added at -196 ${ }^{\circ} \mathrm{C}$. The tube was thawed and shaken producing a red solution. All of the volatile material was removed in vacuo and the tube was transferred to the drybox. The resulting red solid

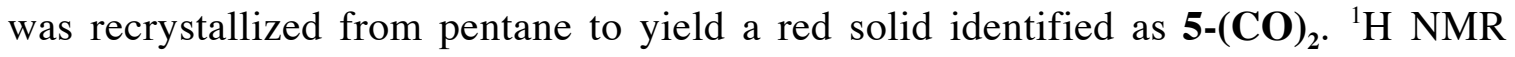
(benzene- $\left.d_{6}\right): \square 1.29\left(\mathrm{~s}, 18 \mathrm{H}, \mathrm{C}_{5} \mathrm{Me}_{4}{ }^{t} B u\right), 1.65\left(\mathrm{~s}, 12 \mathrm{H}, \mathrm{C}_{5} M e_{4}{ }^{t} \mathrm{Bu}\right), 1.83(\mathrm{~s}, 12 \mathrm{H}$, $\left.\mathrm{C}_{5} \mathrm{Me}_{4}{ }^{\mathrm{t}} \mathrm{Bu}\right) .{ }^{13} \mathrm{C}$ NMR (benzene- $\left.d_{6}\right): \square 11.64,14.55,32.92\left(\mathrm{Me} /{ }^{\mathrm{t}} \mathrm{Bu}\right), 34.80\left({ }^{\mathrm{t}} \mathrm{Bu}\right), 103.13$, 106.36, $117.63(\mathrm{Cp}), 267.29$ (CO). IR (pentane): $\square, 1855,1933 \mathrm{~cm}^{-1}(\mathrm{CO})$.

Observation of $\left(\square^{5}-\mathbf{C}_{5} \mathbf{M e}_{4}{ }^{t} \mathbf{B u}\right)_{2} \mathbf{T i}\left(\mathbf{N}_{2}\right)_{2}\left(\mathbf{5}-\left(\mathbf{N}_{2}\right)_{2}\right.$. A React IR cell was charged with 0.023 $\mathrm{g}(0.057 \mathrm{mmol})$ of $\mathbf{5}$ and dissolved in a total volume of $10 \mathrm{~mL}$ of pentane against a flow of dinitrogen. The cell was cooled to $-78{ }^{\circ} \mathrm{C}$ and a spectrum was recorded under a flow of dinitrogen. The temperature of binding was determined based on recording spectra at different temperatures and looking for the N-N stretches. IR (pentane, $-78^{\circ} \mathrm{C}$ ): $\square, 1982$, $2090 \mathrm{~cm}^{-1}\left(\mathrm{~N}_{2}\right)$. 
Preparation of $\left(\square^{5}-\mathrm{C}_{5} \mathbf{M e}_{4}{ }_{4} \mathrm{Pr}\right)_{2} \mathbf{T i C l}(\mathbf{6}-\mathrm{Cl})$. This molecule was prepared in a similar manner to 5-Cl using $2.77 \mathrm{~g}(16.3 \mathrm{mmol})$ of $\mathrm{LiC}_{5} \mathrm{Me}_{4}{ }_{4}^{\mathrm{i}} \mathrm{Pr}$ and $3.01 \mathrm{~g}(8.13 \mathrm{mmol})$ of $\mathrm{TiCl}_{3}(\mathrm{THF})_{3}$. Recrystallization from pentane afforded $2.38 \mathrm{~g}(71.5 \%)$ of 6-Cl. Anal. Calcd for $\mathrm{C}_{24} \mathrm{H}_{38} \mathrm{ClTi}$ : C, 70.33; H, 9.34. Found: C, 69.88; H, 9.18. ${ }^{1} \mathrm{H}$ NMR (benzene- $d_{6}$ ): $\square 1.9\left(\square_{1 / 2}=799 \mathrm{~Hz}\right), 5.7\left(\square \square_{1 / 2}=732 \mathrm{~Hz}\right)$. Magnetic susceptibility (benzene- $\left.d_{6}\right): \square_{\mathrm{eff}}=$ $1.77 \square_{\mathrm{B}}$. EPR (toluene): $g=1.96$.

Preparation of $\left(\square^{5}-\mathbf{C}_{5} \mathbf{M}{ }_{4}{ }_{4}^{i} \mathbf{P r}\right)_{2} \mathbf{T i}(\mathbf{6})$. A $100 \mathrm{~mL}$ round-bottomed flask was charged with $16.88 \mathrm{~g}$ of $0.5 \%$ sodium amalgam and approximately $15 \mathrm{~mL}$ of toluene. With vigorous stirring, $0.300 \mathrm{~g}(0.732 \mathrm{mmol})$ of $\left(\square^{5}-\mathrm{C}_{5} \mathrm{Me}_{4}{ }^{i} \mathrm{Pr}\right)_{2} \mathrm{TiCl}$ was added as a blue toluene solution and the resulting reaction mixture was stirred for two days at ambient temperature. The red solution was filtered through a pad of Celite and the toluene was removed in vacuo leaving a red solid identified as 6. Anal. Calcd for $\mathrm{C}_{24} \mathrm{H}_{38} \mathrm{Ti}$ : C, 76.99; H, 10.23. Found: C, 76.80; H, 9.79. UV-vis (pentane): $\square_{\max }=497,564 \mathrm{~nm} .{ }^{1} \mathrm{H}$ NMR (benzene- $d_{6}$ ): $\square 3.19$ (br s, $2 \mathrm{H}, \mathrm{C}_{5} \mathrm{Me}_{4}{ }_{4}^{i} \operatorname{Pr}$ ), 4.87 (br s, $12 \mathrm{H}, \mathrm{C}_{5} \mathrm{Me}_{4}{ }^{i} \operatorname{Pr}$ ), 45.37 (br s, $12 \mathrm{H}$, $\mathrm{C}_{5} M e_{4}{ }_{4}^{\mathrm{i}} \mathrm{Pr}$ ), 62.70 (br s, $\left.12 \mathrm{H}, \mathrm{C}_{5} M e_{4}^{i} \mathrm{Pr}\right)$. Magnetic susceptibility (benzene- $d_{6}, 23{ }^{\circ} \mathrm{C}$ ): $\square_{\mathrm{eff}}=$ $2.79 \square_{\mathrm{B}}$.

Preparation of $\left(\square^{5}-\mathbf{C}_{5} \mathbf{M} \mathbf{e}_{4}{ }^{i} \mathbf{P r}\right)_{2} \mathbf{T i}\left(\mathbf{N}_{2}\right)_{2}\left(\mathbf{6}-\left(\mathbf{N}_{2}\right)_{2}\right)$. A $20 \mathrm{~mL}$ scintillation vial was charged with $0.15 \mathrm{~g}(0.401 \mathrm{mmol})$ of $\left(\square^{5}-\mathrm{C}_{5} \mathrm{Me}_{4}{ }^{i} \mathrm{Pr}\right)_{2} \mathrm{Ti}$ and a minimal amount of pentane. The vial was cooled to $-35{ }^{\circ} \mathrm{C}$ and after standing at this temperature overnight, large, dark crystals had formed. The crystals were collected and dried in vacuo yielding $0.135 \mathrm{~g}(78.0 \%)$ of 
6-( $\left(\mathbf{N}_{2}\right)_{2}$. Anal Calcd for $\mathrm{C}_{24} \mathrm{H}_{38} \mathrm{~N}_{4}$ Ti: C, 66.97; H, 8.90; N, 13.01. Found: C, 67.41; H, 9.23; N, 12.72. UV-vis (pentane): $\square_{\max }=655 \mathrm{~nm}$. IR (pentane, $-78{ }^{\circ} \mathrm{C}$ ): $\square, 1986,2090 \mathrm{~cm}^{-}$ ${ }^{1}\left(\mathrm{~N}_{2}\right)$.

Preparation of $\left(\square^{5}-\mathrm{C}_{5} \mathrm{Me}_{4}^{\mathrm{i}} \mathrm{Pr}\right)_{2} \mathbf{T i}(\mathrm{CO})_{2}\left(6-(\mathrm{CO})_{2}\right)$. This molecule was prepared in a similar manner to 5-(CO) $)_{2}$ with $0.05 \mathrm{~g}(0.124 \mathrm{mmol})$ of 6 and 1 atm of CO. Recrystallization from pentane afforded $0.038 \mathrm{~g}(76.0 \%)$ of red crystals identified as 6(CO) $)_{2}$. Anal. Calcd for $\mathrm{C}_{26} \mathrm{H}_{38} \mathrm{O}_{2}$ Ti: C, 72.55; H, 8.90. Found: C, 72.46; H, 9.01. ${ }^{1} \mathrm{H}$ NMR (benzene- $d_{6}$ ): $\square 1.16\left(\mathrm{~d}, 10.4 \mathrm{~Hz}, 12 \mathrm{H}, \mathrm{C}_{5} \mathrm{Me}_{4}{ }^{i} \operatorname{Pr}\right), 1.64\left(\mathrm{~s}, 12 \mathrm{H}, \mathrm{C}_{5} M e_{4}{ }^{\mathrm{i}} \mathrm{Pr}\right), 1.72(\mathrm{~s}, 12 \mathrm{H}$, $\left.\mathrm{C}_{5} M e_{4}{ }_{4}^{\mathrm{P}} \mathrm{Pr}\right), 2.55\left(\mathrm{~m}, 2 \mathrm{H}, \mathrm{C}_{5} \mathrm{Me}_{4}{ }^{i} \mathrm{Pr}\right) .{ }^{13} \mathrm{C}$ NMR (benzene- $\left.d_{6}\right): \square 11.30,11.49,23.27$ (Me/iPr), 27.60 ( $\left.{ }^{\mathrm{i}} \mathrm{Pr}\right), 102.72,104.96,114.30$ (Cp), 264.20 (CO). IR (pentane): $\square, 1859$, $1938 \mathrm{~cm}^{-1}(\mathrm{CO})$. 


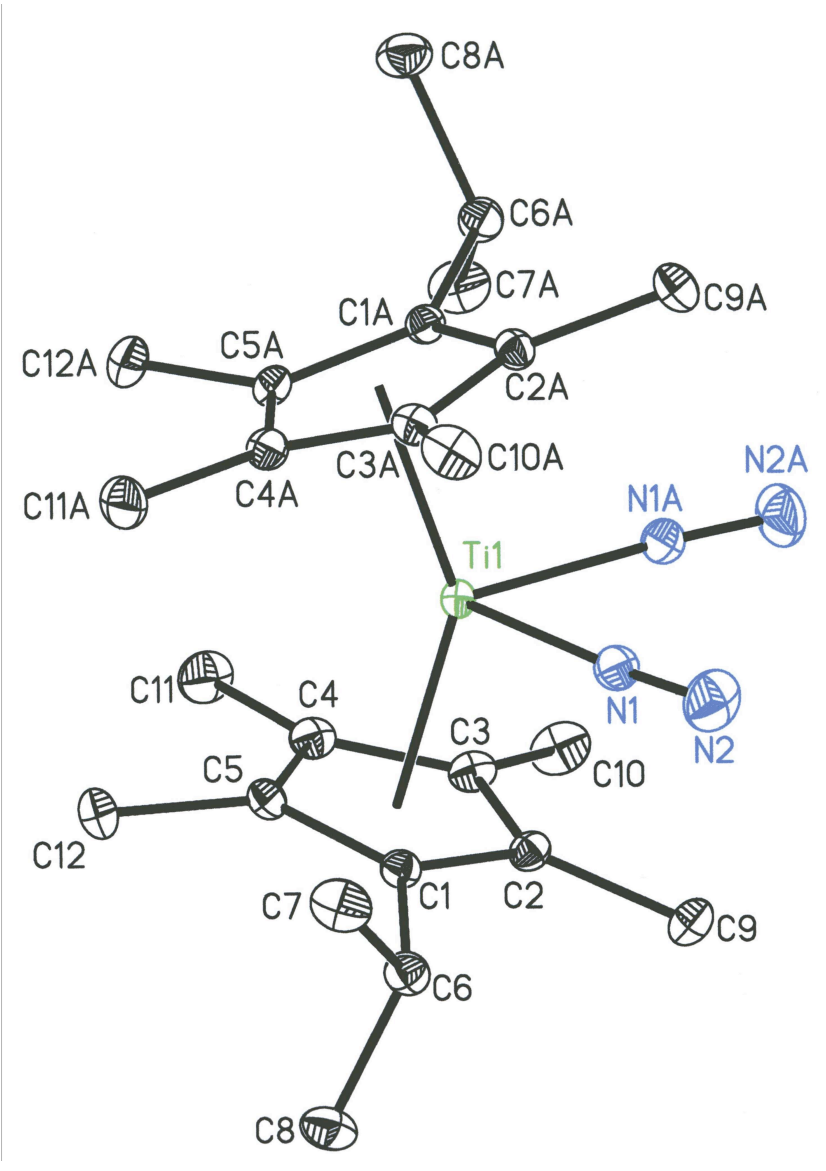

Figure S3. Fully labeled view of the molecular structure of $\mathbf{6}-\left(\mathbf{N}_{2}\right)_{2}$ at $30 \%$ probability ellipsoids. Hydrogen atoms omitted for clarity. 
Table S1. Crystal data and structure refinement for 6-(N $\left.\mathbf{N}_{2}\right)_{2}$.

Identification code

6-( $\left(\mathbf{N}_{2}\right)_{2}$

Empirical formula

$\mathrm{C}_{24} \mathrm{H}_{38} \mathrm{~N}_{4} \mathrm{Ti}$

Formula weight

430.48

Temperature

$173(2) \mathrm{K}$

Wavelength

$0.71073 \AA$

Crystal system

Monoclinic

Space group

Unit cell dimensions

$\mathrm{C} 2 / \mathrm{c}$

$\mathrm{a}=16.4403(13) \AA$

$\square=90^{\circ}$.

$\mathrm{b}=8.5815(7) \AA$

$\square=112.649(2)^{\circ}$.

$c=17.9757(14) \AA$

$\square=90^{\circ}$.

Volume

$2340.5(3) \AA^{3}$

Z

4

Density (calculated)

$1.222 \mathrm{Mg} / \mathrm{m}^{3}$

Absorption coefficient

$0.383 \mathrm{~mm}^{-1}$

$\mathrm{F}(000)$

928

Crystal size

$0.60 \times 0.50 \times 0.15 \mathrm{~mm}^{3}$

Theta range for data collection

2.46 to $30.51^{\circ}$.

Index ranges

$-23<=\mathrm{h}<=23,-12<=\mathrm{k}<=12,-25<=1<=25$

Reflections collected

13707

Independent reflections

$3562[\mathrm{R}($ int $)=0.0537]$

Completeness to theta $=30.51^{\circ}$

Absorption correction

$99.4 \%$

Semiempirical by SADABS

Max. and min. transmission

0.9449 and 0.8030

Refinement method

Full-matrix least-squares on $\mathrm{F}^{2}$

Data / restraints / parameters

$3562 / 0 / 208$

Goodness-of-fit on $\mathrm{F}^{2}$

1.030

Final $\mathrm{R}$ indices [I $>2$ sigma(I)]

$\mathrm{R} 1=0.0428, \mathrm{wR} 2=0.0983$

$\mathrm{R}$ indices (all data)

$\mathrm{R} 1=0.0675, \mathrm{wR} 2=0.1077$

Largest diff. peak and hole

0.338 and -0.296 e. $\AA^{-3}$ 
Table S2. Bond lengths $[\AA]$ and angles $\left[^{\circ}\right]$ for $\mathbf{6}-\left(\mathbf{N}_{2}\right)_{2}$.

\begin{tabular}{|c|c|c|c|}
\hline $\operatorname{Ti}(1)-\mathrm{N}(1) \# 1$ & $2.0428(14)$ & $\mathrm{N}(1)-\operatorname{Ti}(1)-\mathrm{C}(1) \# 1$ & $116.34(5)$ \\
\hline $\operatorname{Ti}(1)-\mathrm{N}(1)$ & $2.0428(14)$ & $C(2)-\operatorname{Ti}(1)-C(1) \# 1$ & $153.01(5)$ \\
\hline $\operatorname{Ti}(1)-C(2)$ & $2.3596(15)$ & $\mathrm{C}(2) \# 1-\mathrm{Ti}(1)-\mathrm{C}(1) \# 1$ & $35.53(5)$ \\
\hline $\operatorname{Ti}(1)-\mathrm{C}(2) \# 1$ & $2.3597(15)$ & $\mathrm{N}(1) \# 1-\mathrm{Ti}(1)-\mathrm{C}(1)$ & $116.34(5)$ \\
\hline $\operatorname{Ti}(1)-\mathrm{C}(1) \# 1$ & $2.3613(15)$ & $\mathrm{N}(1)-\mathrm{Ti}(1)-\mathrm{C}(1)$ & $79.01(5)$ \\
\hline $\operatorname{Ti}(1)-\mathrm{C}(1)$ & $2.3613(15)$ & $\mathrm{C}(2)-\mathrm{Ti}(1)-\mathrm{C}(1)$ & $35.54(5)$ \\
\hline $\operatorname{Ti}(1)-C(5)$ & $2.4039(15)$ & $\mathrm{C}(2) \# 1-\mathrm{Ti}(1)-\mathrm{C}(1)$ & $153.01(5)$ \\
\hline $\operatorname{Ti}(1)-\mathrm{C}(5) \# 1$ & $2.4040(15)$ & $\mathrm{C}(1) \# 1-\mathrm{Ti}(1)-\mathrm{C}(1)$ & $160.97(8)$ \\
\hline $\operatorname{Ti}(1)-\mathrm{C}(3)$ & $2.4160(14)$ & $\mathrm{N}(1) \# 1-\mathrm{Ti}(1)-\mathrm{C}(5)$ & $131.61(5)$ \\
\hline $\operatorname{Ti}(1)-C(3) \# 1$ & $2.4161(14)$ & $\mathrm{N}(1)-\mathrm{Ti}(1)-\mathrm{C}(5)$ & $111.85(6)$ \\
\hline $\operatorname{Ti}(1)-C(4)$ & $2.4291(15)$ & $\mathrm{C}(2)-\mathrm{Ti}(1)-\mathrm{C}(5)$ & $57.87(5)$ \\
\hline $\operatorname{Ti}(1)-\mathrm{C}(4) \# 1$ & $2.4291(15)$ & $\mathrm{C}(2) \# 1-\mathrm{Ti}(1)-\mathrm{C}(5)$ & $148.06(5)$ \\
\hline $\mathrm{N}(1)-\mathrm{N}(2)$ & $1.110(2)$ & $\mathrm{C}(1) \# 1-\mathrm{Ti}(1)-\mathrm{C}(5)$ & $126.71(6)$ \\
\hline$C(1)-C(5)$ & $1.423(2)$ & $\mathrm{C}(1)-\mathrm{Ti}(1)-\mathrm{C}(5)$ & $34.75(5)$ \\
\hline $\mathrm{C}(1)-\mathrm{C}(2)$ & $1.441(2)$ & $\mathrm{N}(1) \# 1-\mathrm{Ti}(1)-\mathrm{C}(5) \# 1$ & $111.85(6)$ \\
\hline$C(1)-C(6)$ & $1.519(2)$ & $\mathrm{N}(1)-\operatorname{Ti}(1)-\mathrm{C}(5) \# 1$ & $131.61(5)$ \\
\hline$C(2)-C(3)$ & $1.427(2)$ & $\mathrm{C}(2)-\operatorname{Ti}(1)-\mathrm{C}(5) \# 1$ & $148.06(5)$ \\
\hline$C(2)-C(9)$ & $1.502(2)$ & $\mathrm{C}(2) \# 1-\mathrm{Ti}(1)-\mathrm{C}(5) \# 1$ & $57.87(5)$ \\
\hline$C(3)-C(4)$ & $1.406(2)$ & $\mathrm{C}(1) \# 1-\mathrm{Ti}(1)-\mathrm{C}(5) \# 1$ & $34.75(5)$ \\
\hline$C(3)-C(10)$ & $1.499(2)$ & $\mathrm{C}(1)-\operatorname{Ti}(1)-\mathrm{C}(5) \# 1$ & $126.71(6)$ \\
\hline$C(4)-C(5)$ & $1.431(2)$ & $\mathrm{C}(5)-\mathrm{Ti}(1)-\mathrm{C}(5) \# 1$ & $94.74(8)$ \\
\hline$C(4)-C(11)$ & $1.504(2)$ & $\mathrm{N}(1) \# 1-\mathrm{Ti}(1)-\mathrm{C}(3)$ & $74.75(5)$ \\
\hline$C(5)-C(12)$ & $1.505(2)$ & $\mathrm{N}(1)-\operatorname{Ti}(1)-\mathrm{C}(3)$ & $110.06(5)$ \\
\hline$C(6)-C(7)$ & $1.529(2)$ & $\mathrm{C}(2)-\operatorname{Ti}(1)-\mathrm{C}(3)$ & $34.75(5)$ \\
\hline \multirow[t]{2}{*}{$C(6)-C(8)$} & $1.532(2)$ & $\mathrm{C}(2) \# 1-\mathrm{Ti}(1)-\mathrm{C}(3)$ & $147.78(5)$ \\
\hline & & $\mathrm{C}(1) \# 1-\mathrm{Ti}(1)-\mathrm{C}(3)$ & $120.81(5)$ \\
\hline $\mathrm{N}(1) \# 1-\mathrm{Ti}(1)-\mathrm{N}(1)$ & $80.18(8)$ & $\mathrm{C}(1)-\mathrm{Ti}(1)-\mathrm{C}(3)$ & $58.03(5)$ \\
\hline $\mathrm{N}(1) \# 1-\mathrm{Ti}(1)-\mathrm{C}(2)$ & $81.41(5)$ & $\mathrm{C}(5)-\mathrm{Ti}(1)-\mathrm{C}(3)$ & $56.96(5)$ \\
\hline $\mathrm{N}(1)-\mathrm{Ti}(1)-\mathrm{C}(2)$ & $77.97(5)$ & $\mathrm{C}(5) \# 1-\mathrm{Ti}(1)-\mathrm{C}(3)$ & $118.32(5)$ \\
\hline $\mathrm{N}(1) \# 1-\mathrm{Ti}(1)-\mathrm{C}(2) \# 1$ & $77.97(5)$ & $\mathrm{N}(1) \# 1-\mathrm{Ti}(1)-\mathrm{C}(3) \# 1$ & $110.06(5)$ \\
\hline $\mathrm{N}(1)-\mathrm{Ti}(1)-\mathrm{C}(2) \# 1$ & $81.41(5)$ & $\mathrm{N}(1)-\operatorname{Ti}(1)-\mathrm{C}(3) \# 1$ & $74.74(6)$ \\
\hline$C(2)-T i(1)-C(2) \# 1$ & $152.95(8)$ & $\mathrm{C}(2)-\mathrm{Ti}(1)-\mathrm{C}(3) \# 1$ & $147.78(5)$ \\
\hline $\mathrm{N}(1) \# 1-\mathrm{Ti}(1)-\mathrm{C}(1) \# 1$ & $79.00(5)$ & $\mathrm{C}(2) \# 1-\mathrm{Ti}(1)-\mathrm{C}(3) \# 1$ & $34.76(5)$ \\
\hline
\end{tabular}




\begin{tabular}{|c|c|c|c|}
\hline $\mathrm{C}(1) \# 1-\mathrm{Ti}(1)-\mathrm{C}(3) \# 1$ & $58.03(5)$ & $\mathrm{C}(6)-\mathrm{C}(1)-\operatorname{Ti}(1)$ & $128.69(10)$ \\
\hline $\mathrm{C}(1)-\mathrm{Ti}(1)-\mathrm{C}(3) \# 1$ & $120.81(5)$ & $\mathrm{C}(3)-\mathrm{C}(2)-\mathrm{C}(1)$ & $107.83(13)$ \\
\hline $\mathrm{C}(5)-\mathrm{Ti}(1)-\mathrm{C}(3) \# 1$ & $118.32(5)$ & $\mathrm{C}(3)-\mathrm{C}(2)-\mathrm{C}(9)$ & $124.21(14)$ \\
\hline $\mathrm{C}(5) \# 1-\mathrm{Ti}(1)-\mathrm{C}(3) \# 1$ & $56.96(5)$ & $\mathrm{C}(1)-\mathrm{C}(2)-\mathrm{C}(9)$ & $127.39(14)$ \\
\hline C(3)-Ti(1)-C(3)\#1 & $174.01(8)$ & $\mathrm{C}(3)-\mathrm{C}(2)-\mathrm{Ti}(1)$ & $74.78(8)$ \\
\hline N(1)\#1-Ti(1)-C(4) & $103.03(5)$ & $\mathrm{C}(1)-\mathrm{C}(2)-\mathrm{Ti}(1)$ & $72.29(8)$ \\
\hline $\mathrm{N}(1)-\mathrm{Ti}(1)-\mathrm{C}(4)$ & $133.56(5)$ & $C(9)-C(2)-\operatorname{Ti}(1)$ & $125.43(11)$ \\
\hline $\mathrm{C}(2)-\mathrm{Ti}(1)-\mathrm{C}(4)$ & $57.36(5)$ & $C(4)-C(3)-C(2)$ & $108.45(13)$ \\
\hline C(2)\#1-Ti(1)-C(4) & $144.98(5)$ & $C(4)-C(3)-C(10)$ & $125.03(15)$ \\
\hline C(1)\#1-Ti(1)-C(4) & $109.63(5)$ & $\mathrm{C}(2)-\mathrm{C}(3)-\mathrm{C}(10)$ & $125.95(15)$ \\
\hline C(1)-Ti(1)-C(4) & $57.75(5)$ & $\mathrm{C}(4)-\mathrm{C}(3)-\mathrm{Ti}(1)$ & $73.64(9)$ \\
\hline C(5)-Ti(1)-C(4) & $34.45(5)$ & $\mathrm{C}(2)-\mathrm{C}(3)-\mathrm{Ti}(1)$ & $70.46(8)$ \\
\hline C(5)\#1-Ti(1)-C(4) & $90.86(5)$ & $C(10)-C(3)-T i(1)$ & $128.57(12)$ \\
\hline C(3)-Ti(1)-C(4) & $33.74(5)$ & $\mathrm{C}(3)-\mathrm{C}(4)-\mathrm{C}(5)$ & $108.21(13)$ \\
\hline C(3)\#1-Ti(1)-C(4) & $140.27(6)$ & $\mathrm{C}(3)-\mathrm{C}(4)-\mathrm{C}(11)$ & $123.97(15)$ \\
\hline $\mathrm{N}(1) \# 1-\mathrm{Ti}(1)-\mathrm{C}(4) \# 1$ & $133.56(5)$ & $\mathrm{C}(5)-\mathrm{C}(4)-\mathrm{C}(11)$ & $126.78(16)$ \\
\hline N(1)-Ti(1)-C(4)\#1 & $103.03(5)$ & $\mathrm{C}(3)-\mathrm{C}(4)-\mathrm{Ti}(1)$ & $72.62(9)$ \\
\hline $\mathrm{C}(2)-\mathrm{Ti}(1)-\mathrm{C}(4) \# 1$ & $144.98(5)$ & $C(5)-C(4)-\operatorname{Ti}(1)$ & $71.82(8)$ \\
\hline $\mathrm{C}(2) \# 1-\mathrm{Ti}(1)-\mathrm{C}(4) \# 1$ & $57.36(5)$ & $C(11)-C(4)-\operatorname{Ti}(1)$ & $130.49(12)$ \\
\hline $\mathrm{C}(1) \# 1-\mathrm{Ti}(1)-\mathrm{C}(4) \# 1$ & $57.75(5)$ & $\mathrm{C}(1)-\mathrm{C}(5)-\mathrm{C}(4)$ & $108.31(13)$ \\
\hline $\mathrm{C}(1)-\mathrm{Ti}(1)-\mathrm{C}(4) \# 1$ & 109.63(5) & $C(1)-C(5)-C(12)$ & $127.50(15)$ \\
\hline C(5)-Ti(1)-C(4)\#1 & $90.86(5)$ & $\mathrm{C}(4)-\mathrm{C}(5)-\mathrm{C}(12)$ & $123.05(15)$ \\
\hline $\mathrm{C}(5) \# 1-\mathrm{Ti}(1)-\mathrm{C}(4) \# 1$ & $34.44(5)$ & $C(1)-C(5)-\operatorname{Ti}(1)$ & $70.99(8)$ \\
\hline $\mathrm{C}(3)-\mathrm{Ti}(1)-\mathrm{C}(4) \# 1$ & $140.27(6)$ & $C(4)-C(5)-\operatorname{Ti}(1)$ & $73.74(9)$ \\
\hline $\mathrm{C}(3) \# 1-\mathrm{Ti}(1)-\mathrm{C}(4) \# 1$ & $33.74(5)$ & $C(12)-C(5)-\operatorname{Ti}(1)$ & $130.71(12)$ \\
\hline $\mathrm{C}(4)-\mathrm{Ti}(1)-\mathrm{C}(4) \# 1$ & $106.58(8)$ & $\mathrm{C}(1)-\mathrm{C}(6)-\mathrm{C}(7)$ & $116.29(14)$ \\
\hline $\mathrm{N}(2)-\mathrm{N}(1)-\mathrm{Ti}(1)$ & $174.16(15)$ & $\mathrm{C}(1)-\mathrm{C}(6)-\mathrm{C}(8)$ & $109.82(14)$ \\
\hline $\mathrm{C}(5)-\mathrm{C}(1)-\mathrm{C}(2)$ & 107.19(13) & $C(7)-C(6)-C(8)$ & $110.24(15)$ \\
\hline$C(5)-C(1)-C(6)$ & $128.27(14)$ & & \\
\hline $\mathrm{C}(2)-\mathrm{C}(1)-\mathrm{C}(6)$ & $123.34(14)$ & \multicolumn{2}{|c|}{ Symmetry transformations used to generate } \\
\hline $\mathrm{C}(5)-\mathrm{C}(1)-\mathrm{Ti}(1)$ & $74.27(9)$ & equivalent atoms: & \\
\hline $\mathrm{C}(2)-\mathrm{C}(1)-\mathrm{Ti}(1)$ & $72.17(8)$ & $\# 1-x, y,-z+3 / 2$ & \\
\hline
\end{tabular}




\section{References}

${ }^{1}$ Pangborn, A.B.; Giardello, M.A.; Grubbs, R.H.; Rosen, R.K.; Timmers, F.J. Organometallics 1996, 15, 1518.

2 a) Schumann, H.; Zietzke, K.; Erbstein, F.; Weimann, R. J. Organomet. Chem., 1996, 520, 265. b) du Plooy, K.E.; du Toit, J.; Levendis, D.C.; Coville, N. J. J. Organomet. Chem., 1996, 508, 231.

${ }^{3}$ Horacek, M.; Gyepes, R.; Cisarova, I.; Polasek, M.;Varga, V.; Mack, K. Collect. Czech. Chem. Commun., 1996, 61, 1307.

${ }^{4}$ Horacek, M.; Kupfer, V.; Thewalt, U.; Stepnicka, P.; Polasek, M.; Mach, K. Organometallics, 1999, 18, 3572.

${ }^{5}$ Bensley, D. M.; Mintz, E. A. J. Organomet. Chem., 1988, 353, 93.

${ }^{6}$ Sur, S. K. J. Magn. Res. 1989, 82, 169. 